\title{
Size Effects on Sensing Properties of ZnO Nanoparticles for Detection of Isoprene
}

\author{
Yunji Park, Ran Yoo, Seungryol Park, JunHo Lee, Hwaebong Jung, Wooyoung Lee* \\ Department of Materials Science and Engineering, Yonsei University, 50 Yonsei-ro, Seodaemun-gu, \\ Seoul, 03722, Republic of Korea \\ *E-mail:wooyoung@yonsei.ac.kr
}

\begin{abstract}
The sensing properties of $\mathrm{ZnO}$ particles have been investigated for the detection of VOCs (Volatile Organic Compounds), in particular, isoprene $\left(\mathrm{C}_{5} \mathrm{H}_{8}\right)$. According to previous studies, size reduction of $\mathrm{ZnO}$ particles was used as one of the methods for maximizing the reactivity. Thus, we compared the reactivity of the $\mathrm{ZnO}$ particles, classified by average particle sizes, $\sim 5$ (so-called quantum dots (QDs)) and $\sim 25 \mathrm{~nm}$ (so-called nanoparticles (NPs)), for the detection of isoprene. Evidently, from the X-ray diffraction characterizations, the $\mathrm{ZnO}$ particles were successfully synthesized by a wet-chemical synthesis method. According to the XPS and BET results, larger number of oxygen vacancies and specific surface area were observed in the $\mathrm{ZnO}$ QDs compared to the NPs. The maximum response to $1 \mathrm{ppm}$ isoprene was $\sim 33$ at $400{ }^{\circ} \mathrm{C}$ for the QDs and $\sim 5$ at $500{ }^{\circ} \mathrm{C}$ for the NPs. The higher sensing response and lower optimal working temperature of the $\mathrm{ZnO}$ QDs can be attributed to the comparably active adsorption sites and enhanced kinetics of the surface reaction from the size effect. Furthermore, the sensing response of $\mathrm{ZnO} \mathrm{QDs}$ to $1 \mathrm{ppm}$ isoprene is superior to previously reported isoprene sensors that are based on semiconducting metal oxides.
\end{abstract}

Key words: gas sensor, $\mathrm{ZnO}$ nanoparticles, isoprene, biomarker, metal oxide semiconductors

\section{Background and Motivation}

The most common method to monitor cholesterol in blood is by an invasive blood cholesterol test. More advanced devices such as selective ion flow tube mass spectrometry (SIFT-MS) or proton transfer reaction mass spectrometry (PTR-MS) have demonstrated high sensitivity, low detection limit and sufficient selectivity for an exhaled breath [1]. However, such devices are hardly applicable in terms of convenience, cost, and efficiency.

In this work, we present a simple method to fabricate a breath analyzer using chemical resistive gas sensors with nanostructured metal oxides. These simple devices have low fabrication cost, compact size, high sensitivity, and sufficient limit of detection [2]. Metal-oxide semiconducting materials have been widely used in gas sensors because of high sensitivity, easy fabrication, and high chemical stability. Among them, $\mathrm{ZnO}$ has been extensively used in gas sensors owing to its high thermal and chemical stabilities, and superior sensing properties [3].

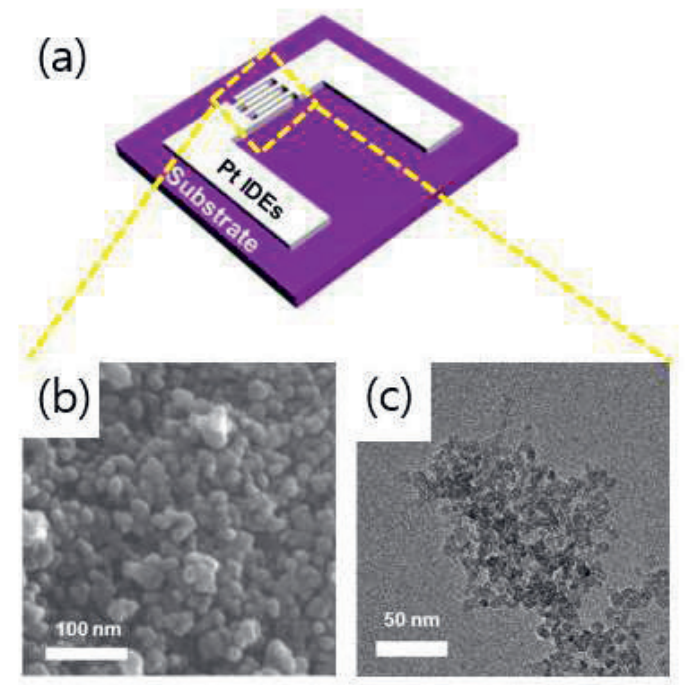

Fig. 1. (a) Schematic illustration of the sensor device; TEM images of actual (b) ZnO NPs and (c) ZnO QDs.

\section{Results and Discussion}

Figure 1(a) shows the conceptual schematic of a sensor device that measures the sensing properties. The micron-scaled $\mathrm{Cr} / \mathrm{Pt}$ electrodes 
were patterned on a $\mathrm{Si} / \mathrm{SiO}_{2}$ substrate. Figures 1 (b) and 1(c) show the TEM images of the assynthesized $\mathrm{ZnO}$ nanoparticles (NPs) and $\mathrm{ZnO}$ quantum dots (QDs), respectively. The TEM images reveal that the ZnO NPs and QDs are in a spherical structure with a diameter of $\sim 25 \mathrm{~nm}$ and $\sim 5 \mathrm{~nm}$, respectively.
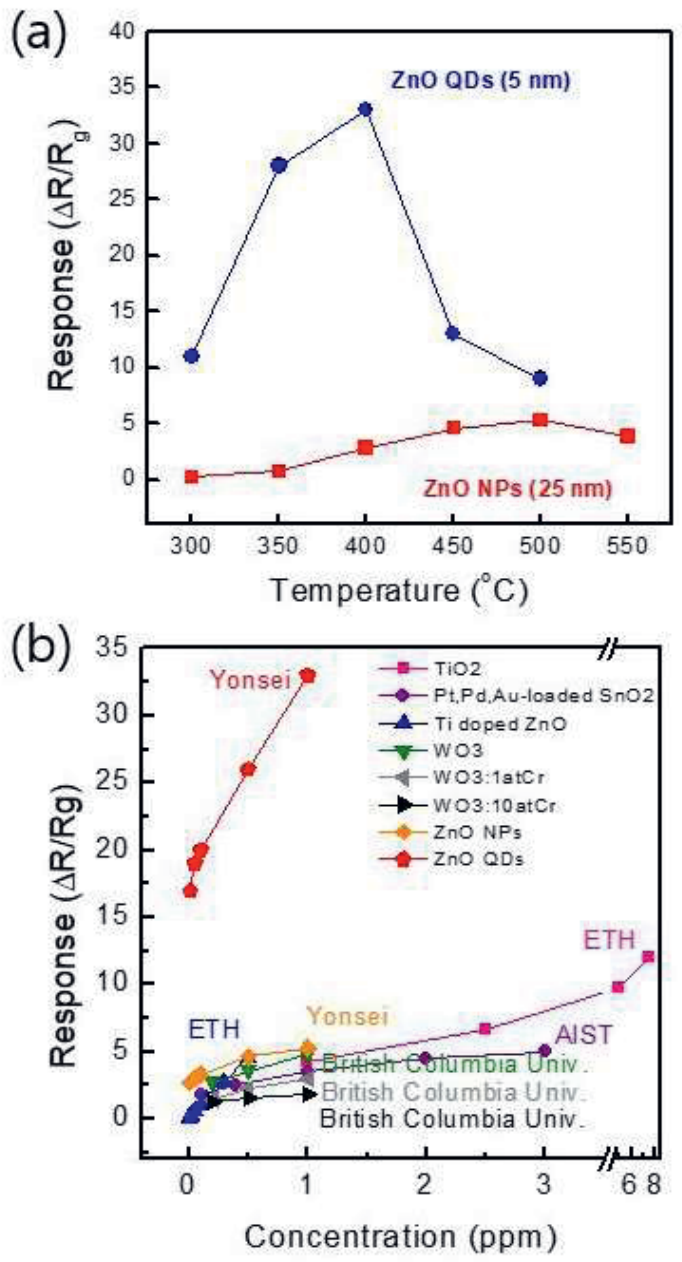

Fig. 2. (a) Maximum sensing response of the $\mathrm{ZnO}$ $N P s$ and $\mathrm{ZnO} Q D$ s toward $1 \mathrm{ppm}$ of isoprene as a function of operating temperature, and (b) sensing response of $\mathrm{ZnO} N P s$ and $\mathrm{ZnO}$ QDs as a function of isoprene concentrations in the range of $0.01-1 \mathrm{ppm}$ at an optimal working temperature.

The sensing properties of ZnO NPs and QDs are showed in Fig. 2. The sensing response for the isoprene gas was defined as $\left(R_{\mathrm{air}}-R_{\mathrm{gas}}\right) / R_{\mathrm{gas}}$ where $R_{\text {gas }}$ and $R_{\text {air }}$ are the resistance of the sensor in an environment containing isoprene and plane air, respectively [3]. The maximum sensing response of the samples to $1 \mathrm{ppm}$ of isoprene are exhibited in Fig. 2(a). As Fig. 2(a) shows, the optimal working temperature was found to be $400^{\circ} \mathrm{C}$ for the $\mathrm{ZnO}$ QDs and $500^{\circ} \mathrm{C}$ for the $\mathrm{ZnO} \mathrm{NPs}$. At the optimum working temperature, the sensing response of the $\mathrm{ZnO}$ QDs ( 33) was much higher than that of the $\mathrm{ZnO}$ NPs ( 5). Figure 2(b) shows the response of the $\mathrm{ZnO}$ NPs and QDs as a function of isoprene concentration at the optimal operating temperature. The data shows a linear increase of the response with isoprene concentration. Ultimately, the $\mathrm{ZnO}$ QDs exhibit superior performance with highest sensitivity value of 16.3 [1-3].
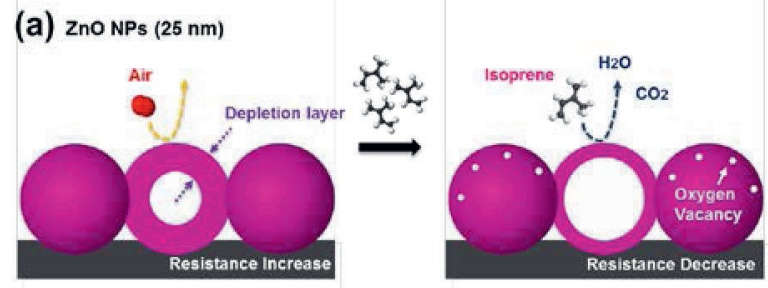

(b) Zno QDs (5 nm)

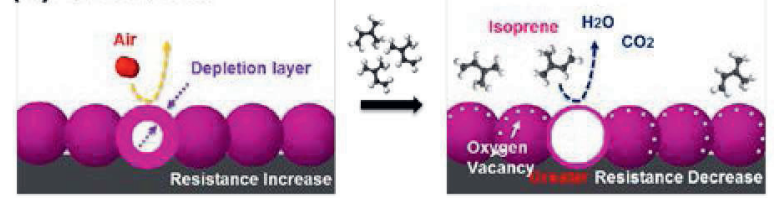

Fig. 3. Schematic diagram of the sensing reaction mechanism of (a) ZnO NPs and (b) ZnO QDs in air and isoprene.

The sensing mechanism for the isoprene detection with the ZnO NPs and the QDs is illustrated in Fig. 3. According to the sensing mechanism, the higher sensing performance is closely related to the increased number of absorbed oxygen ions. Smaller particle size of the ZnO QDs essentially implies larger surface area which consequently produces greater number of reactive sites for isoprene on the surface of the $\mathrm{ZnO}$ QDs. The XPS and BET results revealed that the larger number of oxygen vacancies and specific surface area were observed in the ZnO QDs compared to the NPs. Therefore, the higher sensing response and lower optimal working temperature of the $\mathrm{ZnO}$ QDs can be attributed to the comparably active adsorption sites and enhanced kinetics of the surface reaction from the size effect.

\section{References}

[1] A. T. Guntner, N. J. Pineau, F. Krumeich, and S. E. Pratsinis, Journal of Materials Chemistry B 4, 5358-5366 (2016); doi: 10.1039/c6tb01335j

[2] A. Teleki, S. E. Pratsinis, K. Kalyanasundaram, and P. I. Gouma, Sensors and Actuators B 119, 683-690 (2006); doi: 10.1016/j.snb.2006.01.027

[3] Toshio Itoh, Daiheon Lee, Tomoyo Goto, Takafumi Akamatsu, Noriya Izu, Woosuck Shin, and Toshihiro Kasuga, Sensors and Materials 28, 1165-1178 (2016); doi; 10.18494/SAM.2016.1278

[4] Ran Yoo, Dongmei Li, Sungmee Cho, and Wooyoung Lee, Sensors and Actuators B 254, 1242-1248 (2018); doi: 10.1016/j.snb.2017.07.084 